\title{
Retinal neovascularisation: early contributions of Professor Michaelson and recent observations
}

\author{
ARNALL PATZ \\ From the Wilmer Ophthalmological Institute, Johns Hopkins University and Hospital, Baltimore, \\ Maryland 21205, USA.
}

Summary The late Professor I. C. Michaelson's pioneer contributions to the development and pathophysiology of the retinal vasculature have laid the groundwork for a generation of ophthalmic research scientists to pursue this exciting field of investigation. In more recent studies it has been found that, in diabetic retinopathy, branch vein occlusion, sickle cell retinopathy, and retrolental fibroplasia, retinal neovascularisation follows the development of retinal capillary closure. The capillary closure or nonperfusion has been demonstrated by fluorescein angiography. A working hypothesis to explain the clinical and experimental observations is that these areas of nonperfused retina are ischaemic or hypoxic and liberate a theoretical angiogenic or vasoproliferative substance which stimulates the development of retinal neovascularisation. In postulating this working hypothesis it is important to recognise, firstly, that this hypothesis remains to be proved, and, secondly, that retinal neovascularisation may develop from other stimuli such as intraocular inflammation where retinal ischaemia is not apparent.

This year marks the 30th Anniversary of Professor I. C. Michaelson's completion of his manuscript of the textbook, Retinal Circulation in Man and Animals, which was published in $1954 .{ }^{1}$ This textbook represented a monumental contribution to the understanding of the anatomy and physiology of the retinal circulation. In this issue of the British Journal of Ophthalmology, which is dedicated to the memory of Professor Michaelson, it is appropriate to recognise the significance of his studies which fostered many major research investigations of the retinal vessels.

Professor Michaelson published his first report on the anatomy of the smaller retinal vessels in $1940 .{ }^{2}$ Although interrupted by his military service, he continued these investigations and published in 1948 his classical thesis on 'The mode of development of the retinal vessels and some observations of its significance in certain retinal diseases. ${ }^{3}$

His textbook on the Retinal Circulation in Man and Animals became a classic in the ophthalmic literature and continues to be an important resource reference for contemporary investigators studying the pathophysiology of the retinal vessels. The late Dr Jonas S. Friedenwald, in the foreword to Michaelson's textbook, stated, 'This wide variation in the vascular

Correspondence to Dr A. Patz. pattern supplying the retina is a matter of intrinsic interest from the morphological and morphogenetic point of view, but has bearings on many other fields as well. There is hardly a problem in retinal physiology that cannot be illuminated to some degree by a consideration of the diffusion gradients of metabolites in this tissue. The knowledge that these diffusion gradients are characteristically different in different species, and even in different portions of the retina in some animals, can furnish significant clues to the workers in retinal physiology.' Friedenwald continued by stating, 'Dr. Michaelson's thoughtful, scholarly, and thought-provoking monograph has added much to our knowledge in these matters and should be useful to the morphologist, the physiologist and the clinician concerned with retinal vascular conditions.'

\section{Postulation of angiogenic substances}

The Theory that the developing retina liberates a chemical substance that stimulates retinal vascularisation in the developing retina was first suggested by Michaelson in 1948. Michaelson studied extensively the developing vasculature in the feline retina and concluded that a 'chemical factor' is probably responsible for controlling the growth and development of the retinal vasculature. He suggested that this 
factor had the following properties: (1) the factor is present in the extravascular tissue of the retina; (2) it is present in the gradient of concentration such that it differs in arteriolar and venous neighborhoods; (3) the factor possibly is therefore of a biochemical nature; (4) its action is on the retinal veins predominantly; (5) the factor initiating capillary growth from veins probably determines the distance to which the capillary growth will extend, the initiation and cessation of growth depending on variation and concentration of the factor.

In his discussion of retinal neovascularisation in human retinal diseases, Michaelson stated, 'In the inner eye new-formed vessels may be intra-retinal, pre-retinal, or they may grow into the vitreous chamber. In addition a new formation of vessels may take place on the optic disc. New vessel formation is frequently found in the course of hypertensive and diabetic affections of the retina and in vasculitis retinae of young adults (Eales' disease). Although in venous occlusion of the retina the appearance of new vessel formation is frequently the result of the opening up of pre-existing capillary channels, there is little doubt that in this condition, too, an actual new vessel formation may occur as exemplified in a case recently reported by Michaelson (1948).

'If these cases can be considered as a group they can be seen to have two main features in common. The new vessels are associated nearly always with retinal veins, and secondly, occurring late in these chronic diseases, they are probably a response to changes in retinal and possibly vitreous metabolism.

'It would seem, therefore, that the capacity for new vessel formation possessed by the retinal veins does not disappear. The similarity of circumstances attending the formation of new retinal vessels in development and in pathology, suggests that in disease the formation of new vessels from the retinal veins is a response to the accumulation of an environmental factor in the retina or in the vitreous. This environmental factor elaborated in certain chronic disease processes may be the same as that which is postulated to appear in the developing retina and is capable of initiating budding from retinal veins.

'With this appreciation of the epigenesis of newformed vessels in diabetes and other conditions comes an understanding of the function which those vessels are meant to serve. Just as the metabolic needs of the embryonic retinal tissue demand closer proximity of capillary vessels, so does the disturbed metabolism of certain retinal diseases call for the accession of vessels to insufficiently or non-vascularized situations, intraretinal, pre-retinal or vitreous.'

Michaelson's reference to 'insufficiently or nonvascularized situations' is quite consistent with the current concepts of the association of retinal vascular closure, as demonstrated by fluorescein angiography, with the proliferative retinopathies.

From their brilliant studies on the experimental model of retrolental fibroplasia Ashton and coworkers ${ }^{4}$ postulated the liberation of a 'vasoformative factor' from hypoxic retina. These investigators demonstrated that, following the exposure of young kittens with an incompletely vascularised retina to an increased oxygen environment, vascular closure (vaso-obliteration, the term coined by Ashton and coworkers) occurred. On transfer of the experimental animal from the increased oxygen environment to room air they postulated that the retina nourished by the obliterated vessels became hypoxic, and in response to retinal hypoxia a 'vasoformative factor' was liberated to stimulate the secondary retinal neovascularisation. It was suggested that this presumed vasoformative factor might be related to the substance proposed by Michaelson.

The late George N. Wise provided in his 1955 American Ophthalmological Society thesis ${ }^{5}$ a comprehensive review of 'retinal neovascularization.' He stated that from his findings in retinal vascular disorders that, "these findings strongly suggest the presence of some extra vascular intraretinal unknown factor which stimulates new vessel formation, particularly from the veins.' He referred to this presumed substance as 'factor $x$.' He further suggested that the orderly development of the definitive retinal blood supply suggested a gradient of factor $\mathrm{x}$ in the developing retina. Henkind's Krill memorial lecture, ${ }^{6}$ published in 1978, provides an excellent update on Wise's original review and summarises most of the current concepts of retinal neovascularisation.

\section{Retinal vascular closure and retinal neovascularisation}

Fluorescein angiography has been used extensively during the past 2 decades for the clinical study of retinal vascular disorders, especially the proliferative retinopathies. These include diabetic retinopathy, the neovascularisation following branch vein occlusion, sickle cell anaemia retinopathy, retrolental fibroplasia, and miscellaneous retinal vascular disorders associated with neovascularisation. In addition to its clinical use we also employed fluorescein angiography to investigate the experimental model of retrolental fibroplasia. ${ }^{7}$ We observed that the areas of 'vaso-obliteration' described by Ashton and colleagues represent areas of capillary closure or nonperfusion on fluorescein angiography. These have been observed in the animal model prior to the development of retinal neovascularisation. From these clinical and experimental studies the important role of retinal vascular closure in the course of all of these proliferative retinopathies 


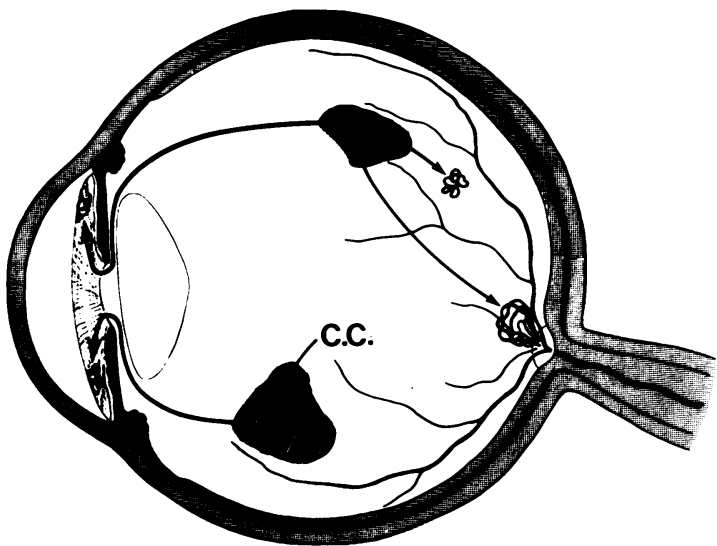

Fig. 1 Retinal capillary closure (C.C.) leads to retinal ischaemia or hypoxia. The ischaemic areas liberate presumed angiogenic substances to stimulate neovascularisation in adjacent areas of the retina, on the optic disc, and on the iris.

has been well documented. It is important to point out that Ashton suggested earlier from his flat retinal preparations of eyes from patients with diabetic retinopathy the important role of retinal capillary closure in diabetes. ${ }^{8}$ His laboratory observations have now been abundantly confirmed in clinical fluorescein studies of patients with diabetic retinopathy.

Examining the neovascularisation that occurs in proliferative diabetic retinopathy, it is important to recognise that retinal neovascularisation is always

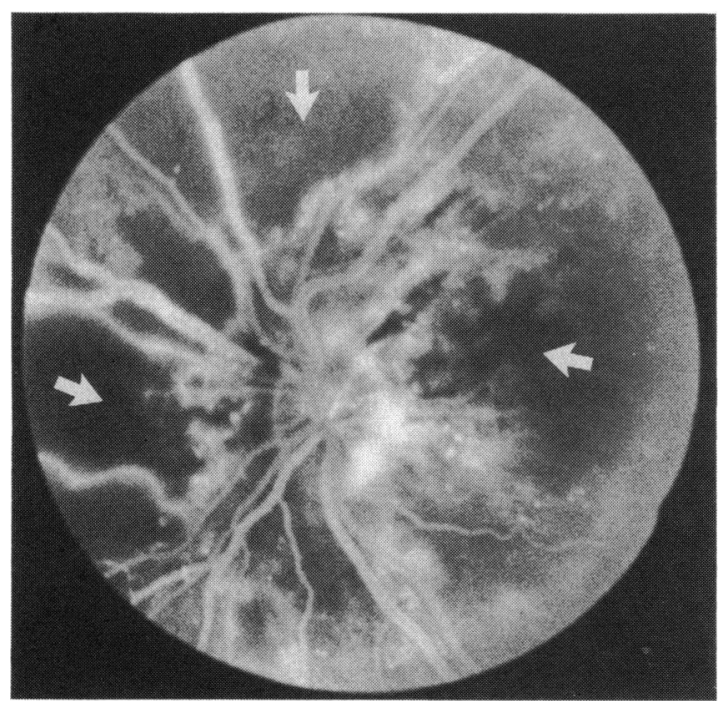

Fig. 2 Fluorescein angiogram on a patient with background retinopathy. Note large areas of capillary nonperfusion (arrows). A few months after this angiogram the patient developed extensive retinal neovascularisation. preceded by the appearance of capillary nonperfusion. Retinal nonperfusion also is noted in branch vein occlusion prior to the development of neovascularisation. Since in branch vein occlusion the time of onset of the vascular closure can frequently be precisely documented, it is of interest that in general the neovascularisation in the patient with a major branch vein obstruction occurs approximately 1 year on average from the onset of the vascular occlusion. In the diabetic patient the greater the degree of capillary nonperfusion, the greater the likelihood for the development of neovascularisation (Fig. 1). Similarly, in branch vein occlusion generally only those patients with large areas of capillary closure have a significant incidence of subsequent retinal neovascularisation.

The clinical and experimental observations in the proliferative retinopathies are consistent with the working hypothesis that retinal ischaemia or hypoxia produced by retinal capillary closure may lead to the production of a biochemical substance, 'angiogenesis factor' or 'vasoproliferative (vasoformative) factor.' Fig. 1 is a schematic representation of the nonperfused (ischaemic) retina liberating the hypothetical angiogenesis substance(s). The angiogenesis material is presumed to stimulate retinal neovascularisation either adjacent to the ischaemic areas in the retina, at the optic disc, or anteriorly on the surface of the iris. In suggesting this hypothesis for the development of retinal neovascularisation it is important to emphasise that this is only a suggested mechanism which is consistent with both the clinical and experimental observations cited above, and the hypothesis remains to be proved (Fig. 2).

It is generally believed that other stimuli may be responsible for the development of retinal neovascularisation. For example, optic disc neovascularisation is a well recognised complication of the inflammatory condition of ocular sarcoidosis. Doxanas and coworkers ${ }^{9}$ have recently reported the complete resolution following the administration of antiinflammatory steroid medication. Another example of inflammation as an apparent source of retinal neovascularisation is the report of Shorb and associates, ${ }^{10}$ who observed several cases of optic disc neovascularisation associated with chronic uveitis, where fluorescein angiography revealed essentially good retinal capillary perfusion.

\section{Photocoagulation and retinal ischaemia}

Panretinal photocoagulation for proliferative diabetic retinopathy has been clearly shown to be beneficial in the Diabetic Retinopathy Study. ${ }^{11}$ Panretinal photocoagulation involves treatment not directed towards the abnormal neovascularisation directly but to the 
surrounding, presumably ischaemic, retinal tissue. Not only is the continued proliferation of the new vessels halted following this treatment, but after panretinal treatment the existing neovascular channels atrophy, leaving little or sometimes no residua. From the histological studies of several investigators we propose, along with other investigators, that the ischaemic inner layers of the retina are altered by panretinal photocoagulation, so that the ischaemic or hypoxic stimulus to retinal neovascularisation is eliminated. It is paradoxical that the major site of laser photocoagulation response is in the outer layers of the retina, yet the ischaemic portion of the retina involves primarily its inner layers. Two related theories taking into account these histopathological observations have been proposed.

(1) Destruction of photoreceptor and pigment epithelial layers increases oxygen available for the inner layers. Weiter and Zuckerman ${ }^{12}$ estimated that the photoreceptors and pigment epithelium consume two-thirds of the total oxygen used by the retina. Destroying these layers should enable a much greater concentration of oxygen to diffuse across the retina from the choriocapillaris to reach the inner ischaemic or hypoxic retinal layers.

(2) Increased oxygen tension in the inner retinal layers after photocoagulation constricts the retinal vessels, especially those feeding the neovascularisation.

Wolbarsht and Landers ${ }^{13}$ measured the retinal tissue oxygen levels at the internal surface of the retina in experimental animals and found that $\mathrm{Po}_{2}$ levels were essentially the same in normal and photocoagulated areas. After mechanically blocking the central retinal artery at the optic disc the $\mathrm{Po}_{2}$ level at the surface of the retina dropped in both the normal and photocoagulated portions of the retina. However, they observed that in normal or nonphotocoagulated retina the $\mathrm{Po}_{2}$ level was lower than in the laser treated retina. These results suggested that panretinal photocoagulation increased the retinal oxygen tension diffusing from the choriocapillaris to the inner layers.

Goldberg ${ }^{14}$ has carefully described the pathogenesis of sickle cell retinopathy. The response of neovascularisation to photocoagulation in sickle cell retinopathy has been recently reported by Rednam $e t$ al.,$^{15}$ and it is similar to that observed in diabetic retinopathy. These investigators applied a scatter pattern of argon laser photocoagulation in the area of vascular occlusion, but deliberately avoided direct treatment to the neovascularisation. In 24 of 28 eyes treated, successful closure of the sea-fan resulted when the neovascularisation was not significantly elevated. The response of the neovascularisation to scatter treatment in the avascular and presumably ischaemic retina in these patients with sickle cell retinopathy is indeed essentially similar to the response of disc neovascularisation and retinal neovascularisation to panretinal photocoagulation treatment in diabetic retinopathy, and the response is consistent with the working hypothesis of ischaemic retina's presumed liberation of angiogenic substances.

\section{Experimental studies on retinal neovascularisation}

In the past decade several laboratories have been extensively involved in basic studies on retinal neovascularisation. Glaser and coworkers $^{16}$ have identified a substance from the excised retina which stimulated the growth of vascular endothelial cells in tissue culture, stimulated endothelial cell migration, and elicited the growth of new vessels on the chick chorioallantoic membrane. This angiogenic substance has now been identified in retinas from rabbits, cows, sheep, dogs, and humans. D'Amore and coworkers ${ }^{17}$ have characterised this retinal angiogenesis substance. A more detailed discussion of these and related angiogenic, as well as angiogenic inhibitor studies, has been recently reported. ${ }^{18} 19$

These studies were aided by a career award from The Seeing Eye, Inc., and supported by a grant from the Helena Rubinstein Foundation.

\section{References}

1 Michaelson IC. Retinal circulation in man and animals. Springfield: Thomas, 1954.

2 Michaelson IC, Campbell ACP. The anatomy of the finer retinal vessels, and some observations on their significance in certain retinal diseases. Trans Ophthalmol Soc UK 1940; 60: 71-112.

3 Michaelson IC. The mode of development of the vascular system of the retina, with some observations on its significance for certain retinal diseases. Trans Ophthalmol Soc UK 1948; 68: 137-80.

4 Ashton N, Ward B, Serpell G. Effect of oxygen on developing retinal vessels with particular reference to the problem of retrolental fibroplasia. Br J Ophthalmol 1954; 38: 397-432.

5 Wise GN. Retinal neovascularization. Trans Am Ophthalmol Soc 1956; 54: 729-826.

6 Henkind P. Ocular neovascularization. The Krill memorial lecture. Am J Ophthalmol 1978; 85: 287-301.

7 Patz A. The role of oxygen in retrolental fibroplasia. Trans Am Ophthalmol Soc 1968; 66: 940-85.

8 Ashton N. Studies of the retinal capillaries in relation to diabetic and other retinopathies. Br J Ophthalmol 1963; 47: 521-38.

9 Doxanas MT, Kelley JS, Prout TE. Sarcoidosis with neovascularization of the optic nerve head. Am J Ophthalmol 1980; 90: $347-51$.

10 Shorb SR, Irvine AR, Kimura SJ, Morris BW. Optic disc neovascularization associated with chronic uveitis. Am J Ophthalmol 1776; 82: 175-8.

11 Diabetic Retinopathy Study Research Group. Preliminary report on effects of photocoagulation therapy. Am J Ophthalmol 1976; 81: 383-96.

12 Weiter JJ, Zuckerman R. The influence of the photoreceptorRPE complex on the inner retina. An explanation for the 
beneficial effect of photocoagulation. Ophthalmology 1980; 87: 1133-9.

13 Wolbarsht ML, Landers MB. The rationale of photocoagulation therapy for proliferative diabetic retinopathy. A review and model. Ophthalmic Surg 1980; 11: 235-45.

14 Goldberg MF. Classification and pathogenesis of proliferative sickle retinopathy. Am J Ophthalmol 1971; 71: 649-65.

15 Rednam KRV, Jampol LM, Goldberg MF. Scatter retinal photocoagulation for proliferative sickle cell retinopathy. Am J Ophthalmol 1982; 93: 594-9.

16 Glaser BM, D’Amore PA, Michels RG, Patz A, Fenselau A.
Demonstration of vasoproliferative activity from mammalian retina. J Cell Biol 1980; 84: 298-304.

17 D'Amore PA, Glaser BM, Brunson SK. Fenselau A. Angiogenic activity from bovine retina. Partial purification and characterization. Proc Natl Acad Sci USA 1981; 78: 3068-72.

18 Glaser BM, Patz A. Ocular neovascularization. Current concepts. In: Little HL, Patz A, Jack RL, Forsham PA, eds. Diabetic retinopathy. New York: Thieme-Stratton, 1983.

19 Patz A. Clinical and experimental studies on retinal neovascularization. XXXIX Edward Jackson memorial lecture. Am Ophthalmol 1982; 94: 715-43. 